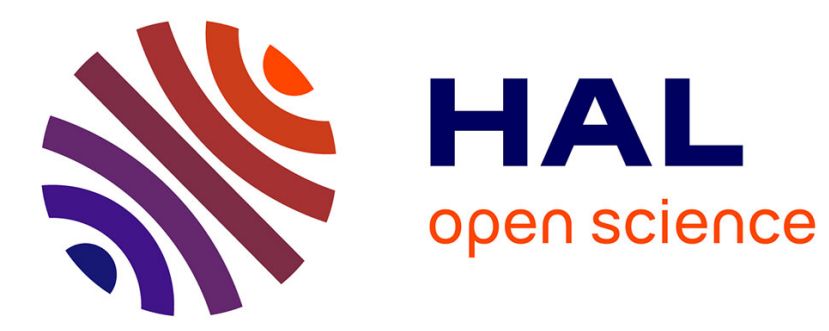

\title{
Estimation of Modal Damping for Structures with Localized Dissipation
}

S Krifa, S Bouhaddi, S Cogan

\section{To cite this version:}

S Krifa, S Bouhaddi, S Cogan. Estimation of Modal Damping for Structures with Localized Dissipation. Special Topics in Structural Dynamics, 2015, Cinccinati, United States. pp.179 - 191, 10.1007/978-3-319-15048-2_17. hal-01512522

\section{HAL Id: hal-01512522 \\ https://hal.science/hal-01512522}

Submitted on 23 Apr 2017

HAL is a multi-disciplinary open access archive for the deposit and dissemination of scientific research documents, whether they are published or not. The documents may come from teaching and research institutions in France or abroad, or from public or private research centers.
L'archive ouverte pluridisciplinaire HAL, est destinée au dépôt et à la diffusion de documents scientifiques de niveau recherche, publiés ou non, émanant des établissements d'enseignement et de recherche français ou étrangers, des laboratoires publics ou privés. 


\title{
Estimation of modal damping for structures with localized dissipation
}

\author{
M. KRIFA, N. BOUHADDI and S. COGAN \\ Applied Mechanics Department, FEMTO-ST - UMR 6174, University of Franche-Comté-24, rue de l'Épitaphe, 25000 Besançon, France
}

\begin{abstract}
Damping plays an important role in bolted joints of assembled structures due to their significant capacity to dissipate energy. The underlying mechanisms of these dissipative phenomena are generally poorly understood and result from contact and friction effects within the joint interfaces. In order to provide useful virtual prototyping tools for reducing response levels, accurate model-based estimation of modal damping is required. The present study employs an energetic method to calculate the loss factor associated with the localized dissipative interfaces of a global linear structure. The current method is based on the concept of the dissipated energy in the interfaces for which the closed-form expression of the loss factor is the ratio between dissipated energy and maximal potential energy, over a cycle of periodic vibration. The aim of this work is to investigate the advantages and drawbacks of this methodology for particular conditions such as: modal projection, localized damping level and model density. Simulated academic examples, where accurate estimations of the exact solutions are available, will be used to illustrate the methodology and to explore the potential difficulties that may arise in more complex industrial applications.
\end{abstract}

Keywords :Bolted joints, loss factor, proportional damping, localized damping, energetic method

\section{Introduction}

The study of the dissipation in the joints has been the subject of several studies [1]. However, the prediction of damping in structures assembled from the design phase started since a decade [2,3]. Bolted joints are responsible for the major part of the energy dissipation in assembled structure [2]. Constitutive modeling of mechanical joints is an important part of predictive dynamic modeling of joints structures because joints are often the dominant source of energy dissipation and vibration damping in those structures. For very expensive components that are safety critical, predictive dynamic modeling is necessary. In order to predict modal damping in an assembled structures two kinds of methods exist in the literature : experimental methods [4] and calculating methods [5]. Afterwards we will only be interested in the calculation methods. Mechanical engineers often use proportional damping assumption for vibration calculations, even if the structures have a configuration of localized damping. The modal assumption risks to not respect physical properties of the dynamic system. This can causes vibration amplitudes differences and modal damping errors when comparing the proportional model with the real model [7]. The purpose of this paper is to investigate the limitations of the energetic method when predicting modal damping for localized structures. Therefore, this paper is structured as follows : section 2 presents the energetic method to estimate modal damping and section 3 illustrates the prediction level of this methodology and its validity domain on academic examples.

\section{Energetic method}

To estimate the damping with localized dissipation in bolted joints of an assembled structures when the eigenmodes are well separated, we can use state space method and the energetic method. In this work we describe just the second method. But results of these two methods are taken into account to calculate modal damping of proposed academic examples.

*Corresponding author mohamed.krifa@femto-st.fr 
The present study employs this approach to calculate the loss factor associated with the localized linear interfaces of a globally linear structure. In practice, the response of the system is not calculated explicitly due to the high computational burden. When assuming a linear and dissipative problem of assembled structures, the discrete form of the damped vibration problem may be governed by the equation :

$$
M \ddot{y}(t)+C \dot{y}(t)+K y(t)=0
$$

where $\mathrm{K}, \mathrm{M}$ and $\mathrm{C}$ are respectively the stiffness, mass and damping matrices, $y$ is the vector of the external loads.

The objective of the energetic method is to determine the damping factor corresponding to each vibration mode of the structure. It is based on the concept of the dissipated energy in the interfaces for which the close form expression of the loss factor is the ratio between dissipated energy and maximal potential energy, over a cycle of periodic vibration. as shown in this relation :

$$
\xi_{\nu}=\frac{1}{4 \pi} \frac{E_{\nu}^{d i s s}}{E_{\nu}^{d e f}} ; \nu=1,2, \ldots ., n
$$

where $E_{\nu}^{\text {diss }}$ and $E_{\nu}^{\text {def }}$ are respectively the dissipated energy and maximal potential energy. In the following we will present the necessary formulas for calculating these energies.

The estimation of the dissipated and maximal potential energies requires an accurate characterization of the response levels of the system and the latter remain an approximation since they depends a priori on a knowledge of the different dissipation mechanisms. So, dissipated energy is calculated by the following expression :

$$
E_{\nu}^{d i s s}=\int_{0}^{T} \dot{y}(t)^{T} f_{c}(t) d t
$$

Where

- $T=\frac{2 \pi}{\omega_{\nu}}$ the cycle of periodic vibration

- $f_{c}(t)=C \dot{y}(t)$ : dissipated force

- $\dot{y}(t)=\operatorname{Re}\left(j \omega_{\nu} y\left(\omega_{\nu}\right) e^{j \omega_{\nu} t}\right)$ the velocity of harmonic response

Potential energy is calculated as follow :

$$
E_{\nu}^{\text {def }}=\frac{1}{2}\left\{y\left(w_{\nu}\right)\right\}^{T} K\left\{y\left(w_{\nu}\right)\right\}
$$

where $y\left(w_{\nu}\right)$ is the frequency response of the system.

We distinguish to cases : the proportional damping and localized damping. In the first case the frequency response of the system is equal to

$$
y\left(w_{\nu}\right)=\sum_{\nu=1}^{n} \phi_{\nu} q_{\nu}
$$

If the response is projected on a single mode of vibration then equation (5) becomes

$$
y\left(\omega_{\nu}\right)=\phi_{\nu} q_{\nu}
$$

where $\phi_{\nu}$ and $q_{\nu}$ are respectively the eigenmode and modal amplitude corresponding to eigenfrequency $\omega_{\nu}$. The exacte frequency response is equal to :

$$
y(\omega)=\left(K+j \omega C-\omega^{2} M\right)^{-1} \times f
$$

In the case of proportional damping, if the eigenmodes $\phi_{\nu}$ are orthonormalized with respect to the mass matrix M, i.e. $\phi_{k}^{T} M \phi_{l}=\delta_{k l}$, so from equations (4) and (5) the total strain energy can be derived in the form :

$$
E_{\nu}^{\text {def }}=\frac{1}{2} \sum_{\nu=1}^{n} \omega_{\nu}^{2} q_{\nu}^{2}
$$


If the damping is assuming to be hysteretic; i.e.

$$
C=\frac{\eta}{\omega} K
$$

where $\eta$ is the unknown loss factor. One can easily show that at frequency $\omega_{\nu}$, the modal damping $q_{\nu}$ can be expressed in fonction of $\xi_{\nu}$ by :

$$
q_{\nu}=-j \frac{\phi_{\nu} f}{\omega_{\nu}^{2} \eta_{\nu}}=-j \frac{\phi_{\nu} f}{2 \omega_{\nu}^{2} \xi_{\nu}}
$$

Where : $\eta_{\nu}=2 * \xi_{\nu}$. Equation (10) leads to the amplitude $q_{\nu}$, from which one can determine the energy dissipated in each joint. Hence, the energy dissipated in all joint leads to the estimated value of the total damping factor :

$$
\xi_{\nu}^{\text {joints }}=\frac{1}{4 \pi} \frac{E^{\text {diss }}}{E^{\text {def }}}
$$

\section{Advantages and limitations of the energetic method}

The energetic method proposed has been implemented on a demonstrator as a model in Matlab. Three numerical simulations have been performed in order to highlight the validity domain of energetic method. The goal of this part consists in studying the influence of the following parameters on the accuracy of this method: modal projection effect, localized damping level and modal density when predicting modal damping.

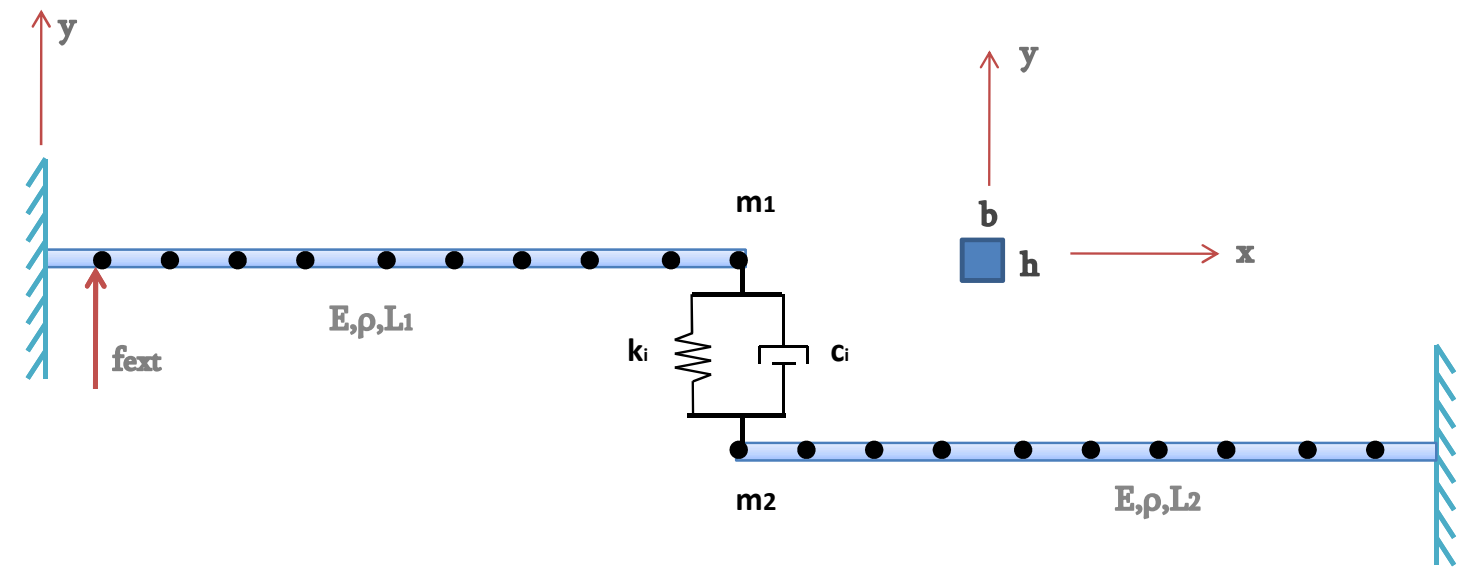

Figure 1: Double-beam with simple bending

Each component is discretized into ten 2D beam finite elements (2 DOFs per node), so the full model has 40 DOFs. Two lumped masses $(\mathrm{m} 1, \mathrm{~m} 2)$ are fixed at the free end of the beams. The excitation force is applied to node number 1 of the first beam. This model represents two structures assembled by a bolting joint. Simulation data are summarized in the tables 1 . The joint is represented by a linear lumped model composed of spring $k_{i}$ and a viscous damper $c_{i}$ elements.

The dissipation force is expressed in terms of the velocity by the equation:

$$
f_{c}(t, \dot{u})=c_{i} \times \Delta \dot{u}
$$

The modal damping results calculated with three methods are illustrated on Figures 2: The reference method is, in this case, the state space method [6], the methods EM1 and EM2 are the variants of the Energetic Method EM respectively with mode projection on truncated modal sub-basis using equation (6) and with modal projection on a single mode of 
Table 1: Physical and geometric properties of the structure / Inerface parameters

\begin{tabular}{llllll}
\hline & & & & & \\
$E$ & Steel's Young modulus $(G P a)$ & 210 & $k_{i}$ & Linear stiffness $(\mathrm{N} / \mathrm{m})$ & $10^{5}$ \\
$\rho_{s}$ & Steel density $\left(\mathrm{kg} / \mathrm{m}^{3}\right)$ & 7800 & $c_{i}$ & Viscous damper $(\mathrm{N} /(\mathrm{m} / \mathrm{s}))$ & 20 \\
$h$ & Beam thikness $(\mathrm{m})$ & $210^{-2}$ & $m_{1}$ & Mass $(\mathrm{Kg})$ & 0.2 \\
$b$ & Beam width $(\mathrm{m})$ & $210^{-2}$ & $m_{2}$ & Mass $(\mathrm{Kg})$ & 0.2 \\
$L_{1}$ & Beam first length $(\mathrm{m})$ & 0.6 & & & \\
$L_{2}$ & Beam second length $(\mathrm{m})$ & 0.5 & & & \\
$\nu$ & Steel's Poisson ratio & 0.3 & & & \\
\hline
\end{tabular}

interest using equation (7). The method EM1 predicts the modal damping with unacceptable errors: for mode 2 (19\%), $4(55 \%)$ and 6(>80\%). Whereas for the method EM2 these error levels drop significantly to reach 0.06 and 0.07 respectively for modes 2 and 4 . This improvement is due, in this case, at the projection in a single mode of the forced response of the system.

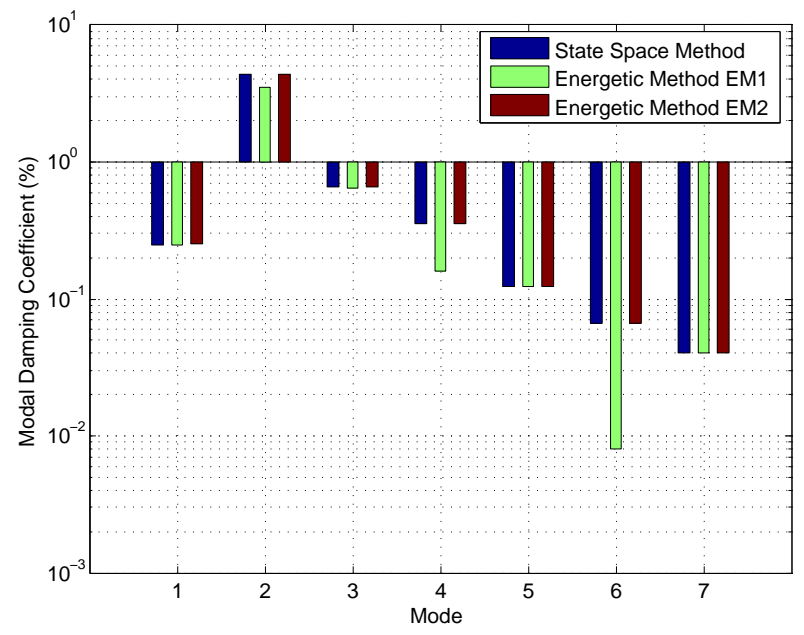

Figure 2: Comparaison of estimated modal damping

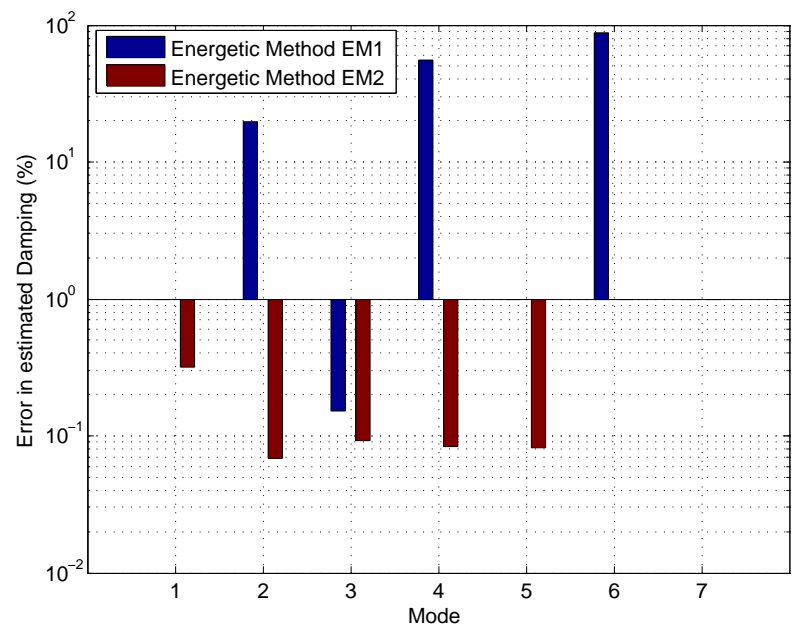

Figure 3: Error in estimating modal damping

Results of damping errors found between the energetic method EM1 and EM2 versus the reference method (figure 3) are expected if one takes into account the contribution of neighboring terms before calculating the modal damping coefficients. The frequency response of the system (figure 1) when looking for a modal damping can be expressed by :

$$
y(\omega)=\sum_{\nu=1}^{n} \phi_{\nu} \frac{\phi_{\nu}^{T} f^{e x t}}{\omega_{\nu}^{2}-\omega^{2}+2 j \omega \omega_{\nu} \xi_{\nu}}=\sum_{\nu=1}^{n} \phi_{\nu} \times q_{\nu}
$$

where $q_{\nu}$ is the contribution of mode $\nu$. The coefficient $\left(\frac{q_{i}}{q_{j}}\right)$ presents the ratio of the contribution of mode $i$ relative to mode $j$. We can thus define a theoretical coefficient of the sum of neighboring mode contribution on single mode $j$ as follows :

$$
\gamma_{j}=100 \times \frac{\sum_{i \neq j} q_{i}{ }^{2}}{q_{j}{ }^{2}}
$$

Figure 4 presents the variation of damping errors with respect to the variation of the sum of neigbouring mode contribution $\gamma_{j}$.

When $\gamma$ increases for one mode, the damping error of this mode increases too. For this case modes 2,4 and 6 have the biggest neighboring mode contribution and the biggest damping error values. In the reminder of this study, we will use the dynamic responses with projection on a single mode as this generates better results in the prediction of the modal damping coefficients. 


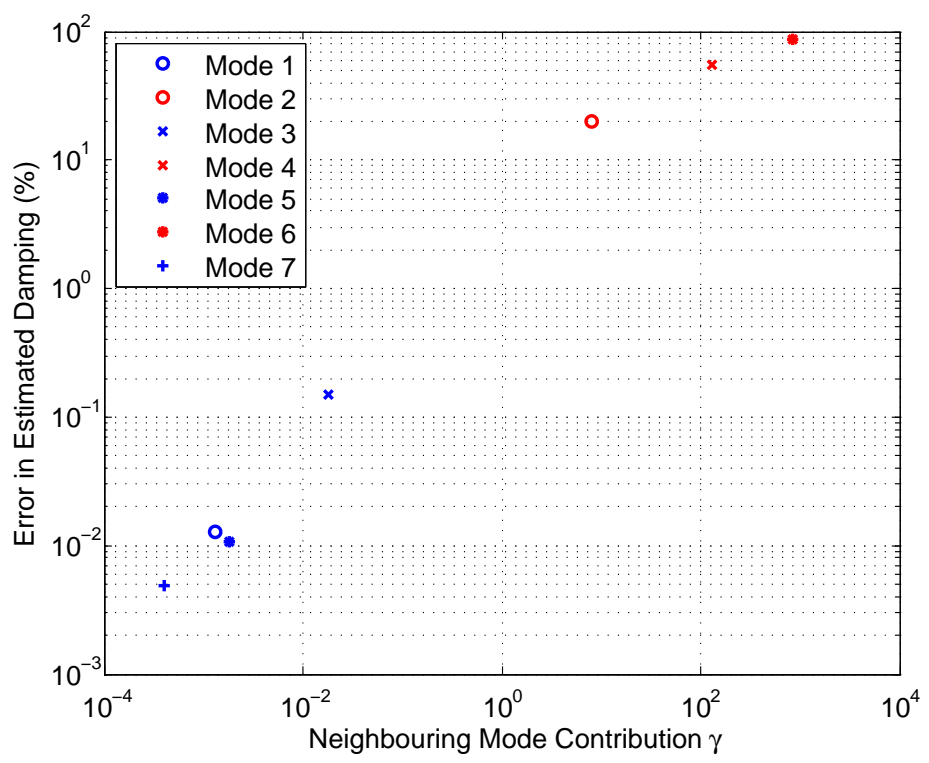

Figure 4: Modal participation of energetic method EM1

\subsection{Effects of the localized damping level}

In this section, we are interested in studying the effect of the localized damping level (figure 1) on damping prediction by the Energetic Method EM2. So, six numerical simulations are performed by increasing the viscous damping $\left(c_{i}=\right.$ $10,20,30,40,50$ and $60 \mathrm{Ns} / \mathrm{m}$ ). When localized damping value $c_{i}$ increases as if the dissipated energy increases. Figure 5 illustrates the evolution of relative error obviously for modes 1 and 2, which is calculated between the energetic method EM2 and the reference method. Figure 5 represents also the curves rise for both modes 1 and 2 when localized damping value increases. For other modes we notice the same curve shape but with lower levels of error.

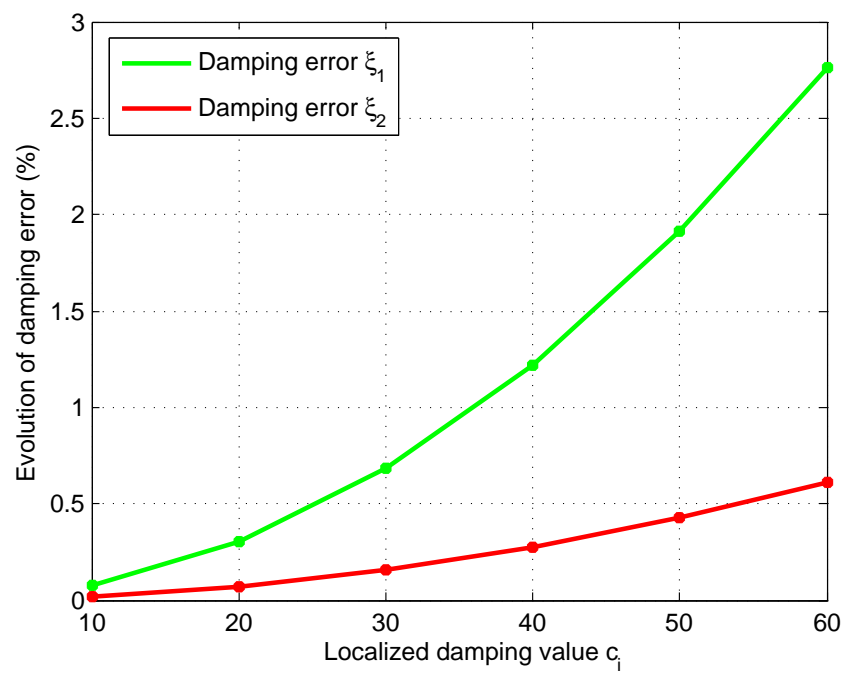

Figure 5: Infuence of the degree of dissipated energy at the interface

When $c_{i}$ increases, for example when $c_{i}=60 \mathrm{~N} /\left(\mathrm{m} / \mathrm{s}\right.$ ), damping value $\xi_{2}$ goes up $10 \%$ and attains a peak of $13 \%$ (figure 6). Beyond this modal damping level, damping error increases to unacceptable values (figure 7). The reference method (state space method) may not give good results when $c_{i}$ exceeds a specific modal damping level.

The raison is that state space method is based on the assumption described by the following equation

$$
s_{\nu}=-\xi_{\nu} \omega_{\nu}+j \omega_{\nu} \sqrt{1-\xi_{\nu}^{2}}, \xi_{\nu}<<1
$$




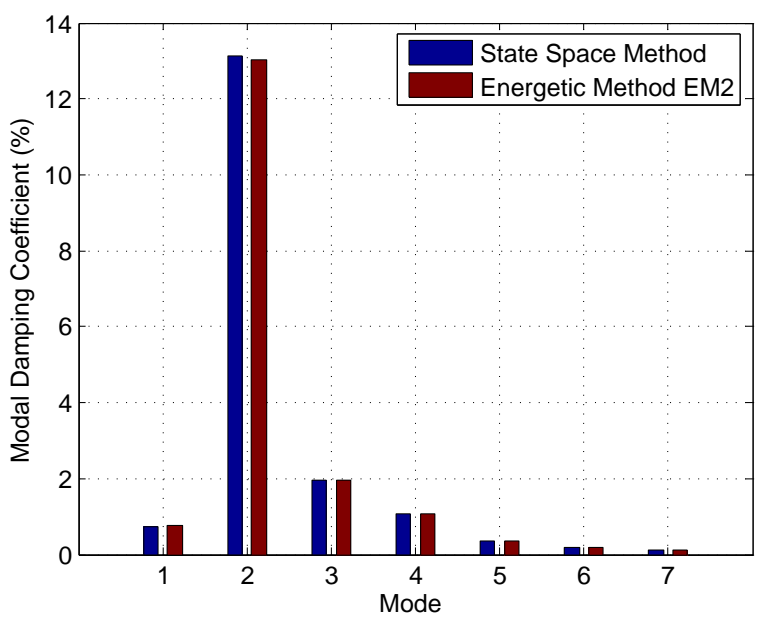

Figure 6: Comparaison of estimated modal damping

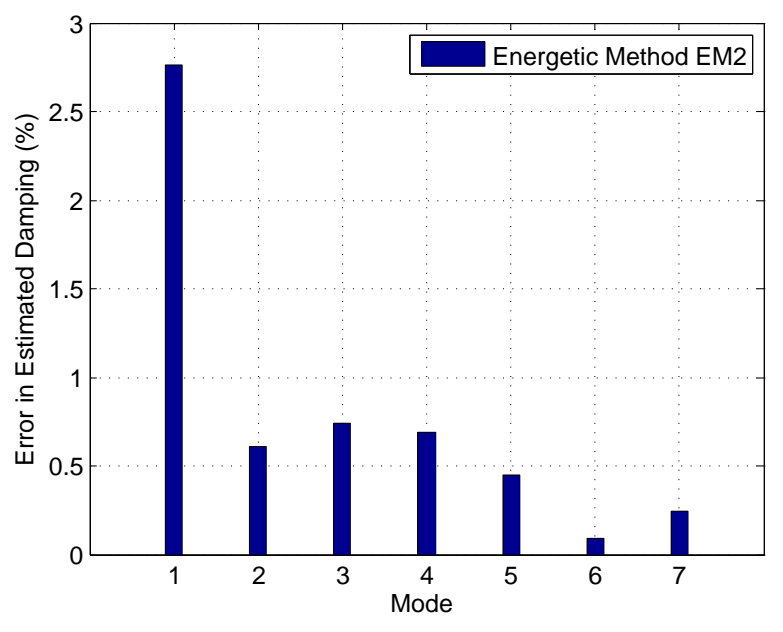

Figure 7: Error in estimating modal damping

Where $s_{\nu}$ is the eigensolution of $\nu^{t h}$ mode $; j^{2}=-1$. So to evaluate the quality of the estimated modal damping by the energetic method, and the previous assumption is valid only with low modal damping values. In the following, we compare the frequency responses functions FRFs of the four methods (energetic method EM1, energetic method EM2, state space and exact method). The exact method consists in the calculation of the FRFs by direct inversion of the full impedance matrix at each frequency (equation (7)). The FRFs computing by the energetic method(EM1, EM2) use a truncated basis of ten modes contained in the frequency band $\left(0,2 \times f^{\max }\right), f^{\max }=2000 \mathrm{~Hz}$ is the maximum frequency of interest.

To calculate FRFs level error in (\%) between two responses $y_{1}$ and $y_{2}$ in the frequency band of interest $[0,2000 \mathrm{~Hz}]$, we use the following expression :

$$
\varepsilon_{y}=100 \times \frac{\left\|y_{2}-y_{1}\right\|}{\left\|y_{1}\right\|}
$$

For State space method, the FRF comparaison with exact method gives almost similar results (figures 8) when $c_{i}=$ $20 \mathrm{Ns} / \mathrm{m}$. But it don't gives the same amplitude levels for high level of viscous damping, for exemple when $c_{i}=60 \mathrm{Ns} / \mathrm{m}$ (figures 9). That's way in the following we will use exact method as a reference instead of state space method, especially when dealing with high damping level.

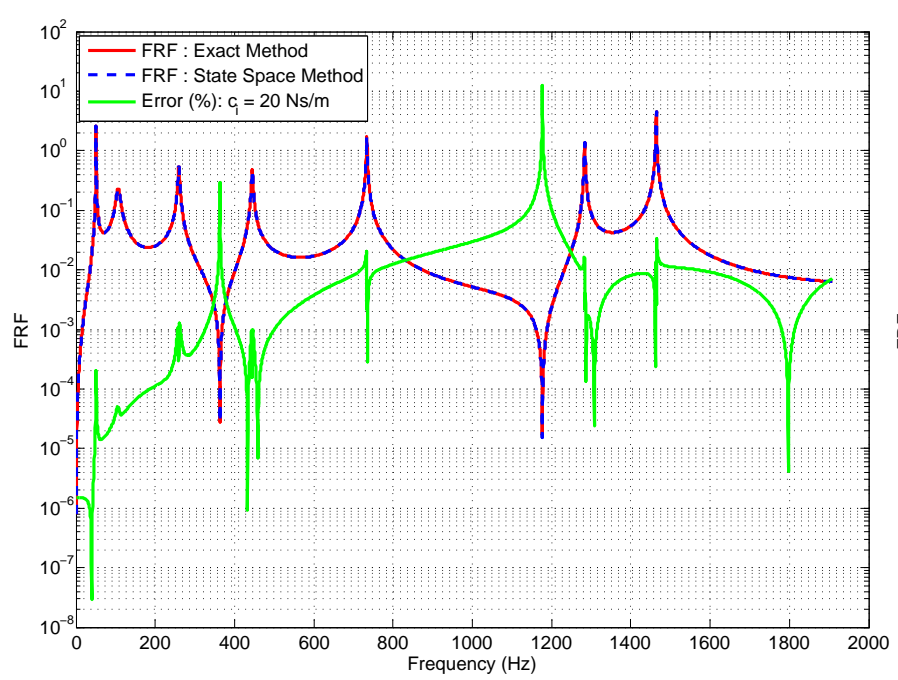

Figure 8: FRFs error: State space vs Exact method $-c_{i}=$ $20 N s / m$

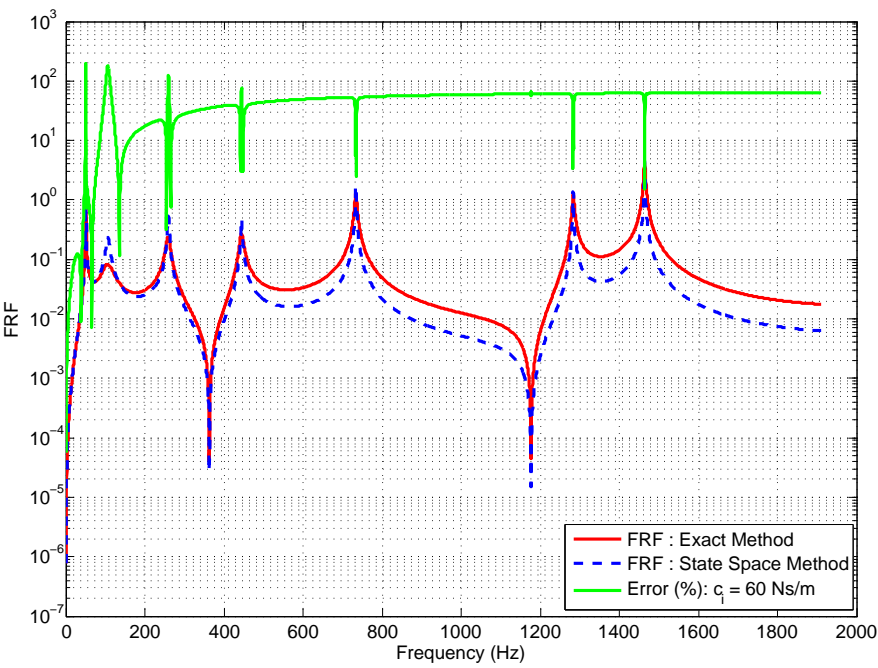

Figure 9: FRFs error: State space vs Exact method $-c_{i}=$ $60 N s / m$

Figures 10 illustrates that the method EM2 estimates the damping factor with good accuracy for low damping level. But, Figure 11 shows that this method is not valid for high damping value. 


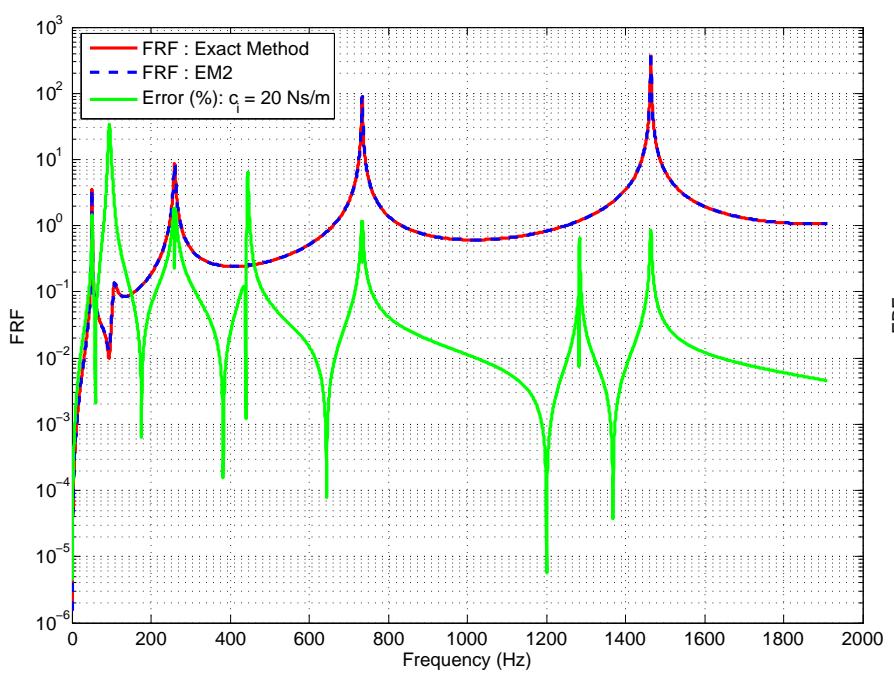

Figure 10: FRFs error: EM2 vs Exact Method $-c_{i}=$ $20 \mathrm{Ns} / \mathrm{m}$

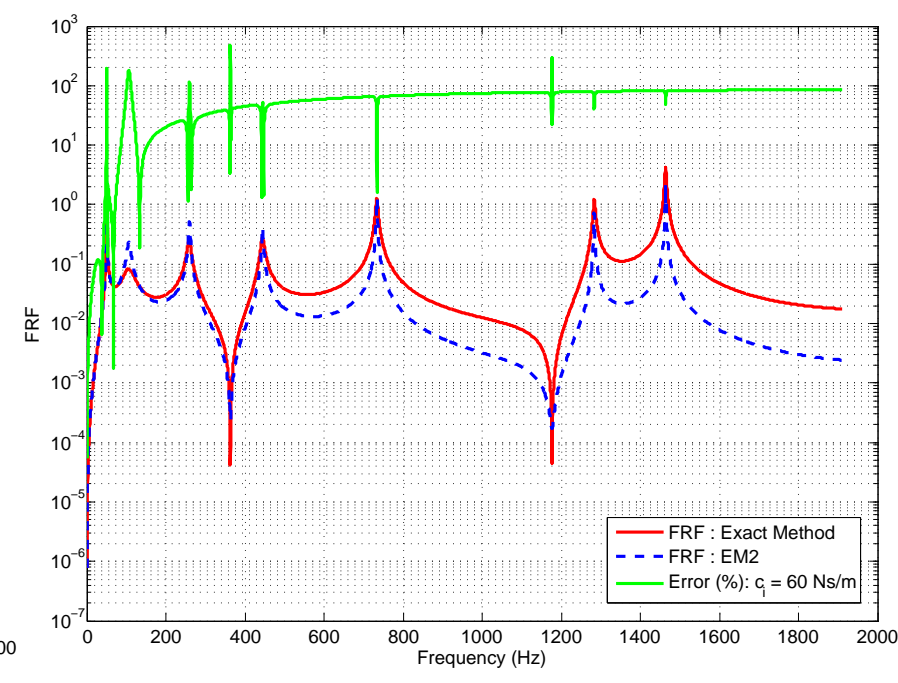

Figure 11: FRFs error: EM2 vs Exact Method $-c_{i}=$ $60 \mathrm{Ns} / \mathrm{m}$

As studied in section 3.1 The method EM1 doesn't give good results when comparing with exact method for both cases $\left(c_{i}=20 N s / m\right.$ and $\left.c_{i}=60 N s / m\right)$, (figures 12 and 13).

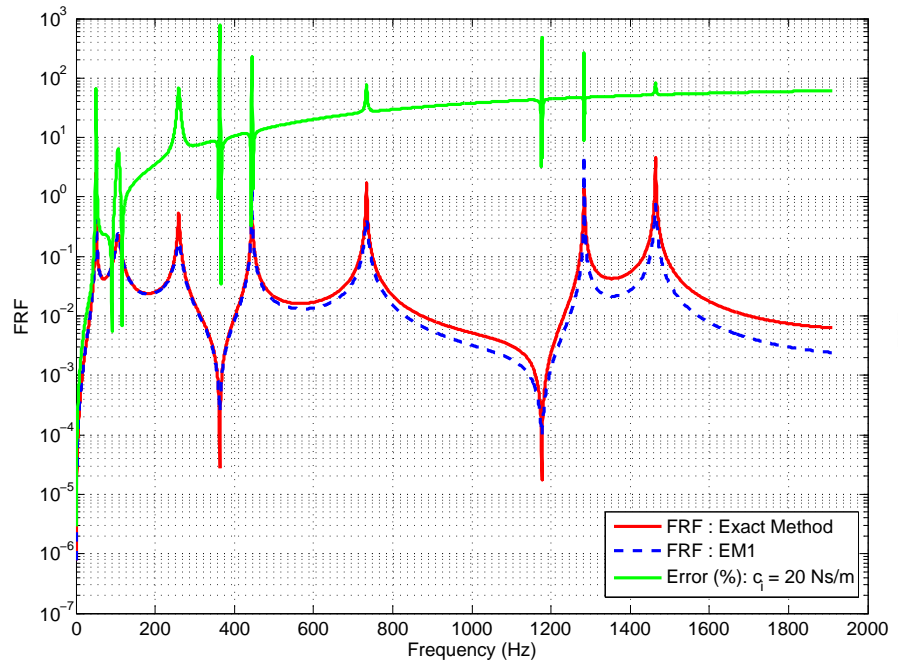

Figure 12: FRFs error: EM1 vs Exact Method $-c_{i}=$ $20 \mathrm{Ns} / \mathrm{m}$

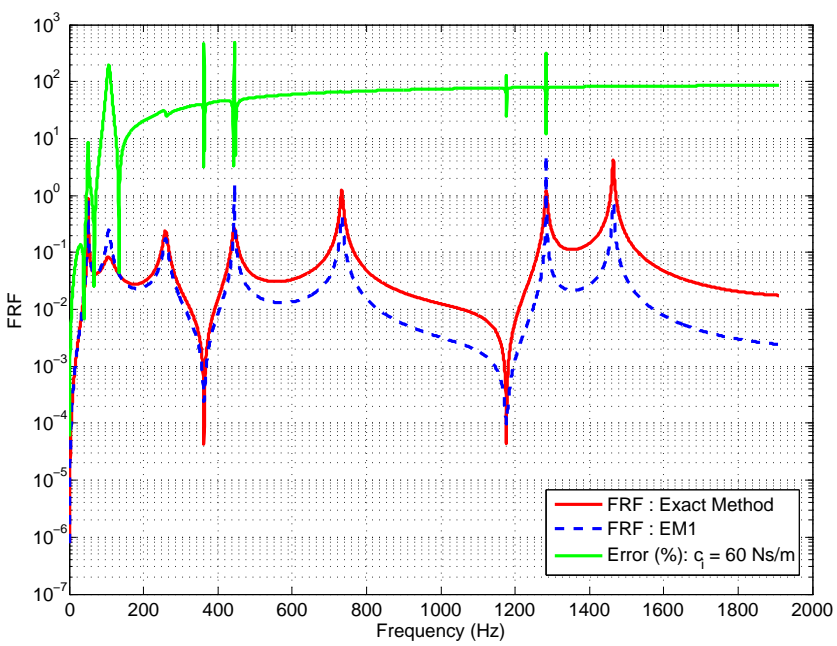

Figure 13: FRFs error: EM1 vs Exact Method $-c_{i}=$ $60 \mathrm{Ns} / \mathrm{m}$

So in the rest of this work we will just investigate the method EM2 in the aim to know it's limits for the specific condition : the influence of modal density. 


\subsection{Influence of modal density}

In th

triple

the $\mathrm{F}$

respe

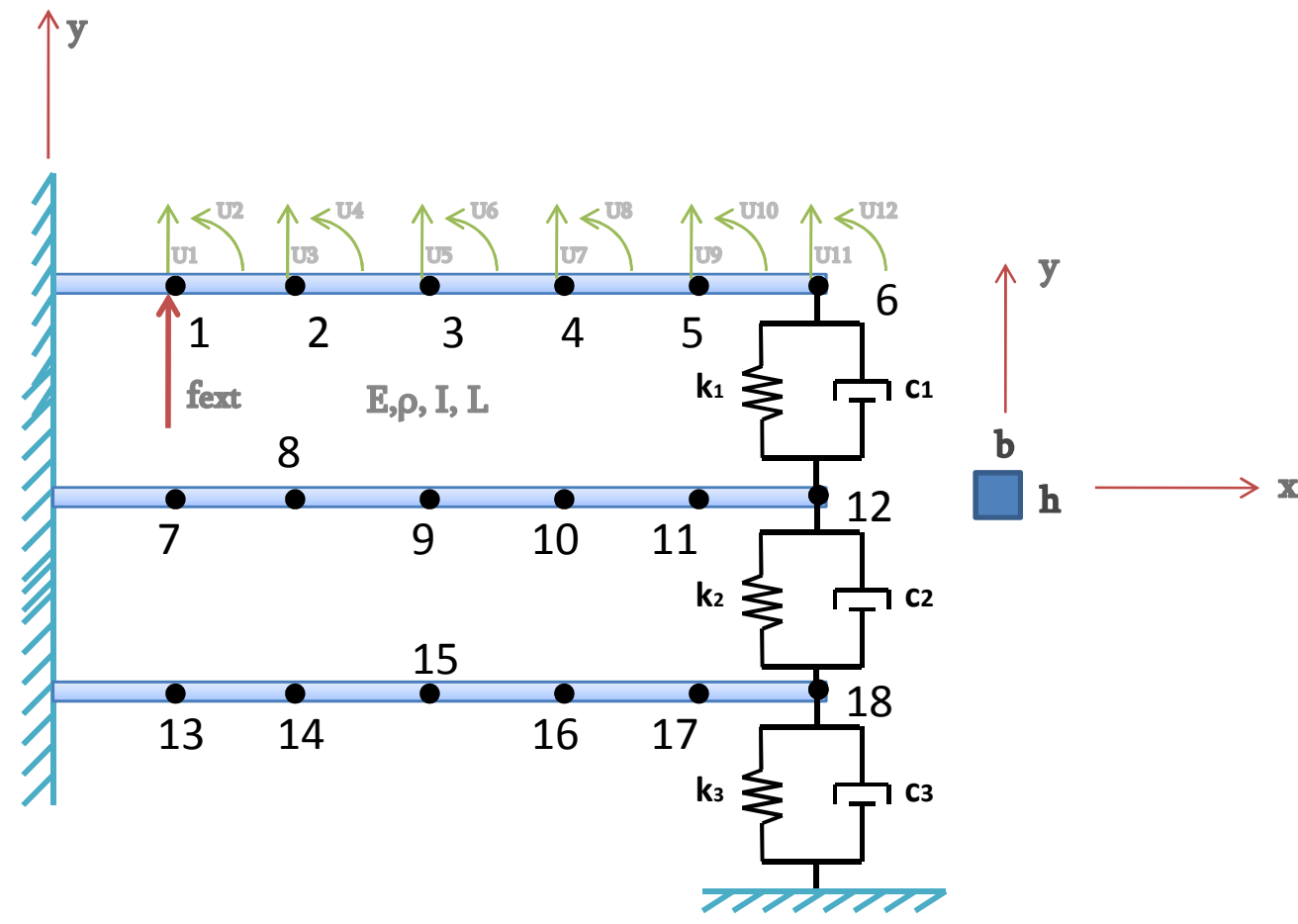

Figure 14: Triple-beam with simple bending

Each interface consists of a stiffness $k_{i}$ and a linear damper $c_{i}$. The simple bending excitation is applied to the node number 1. the used parameters are performed in table 2. Hasselman [8] proposed the following condition of decoupling modes :

Table 2: Physical and geometric properties of the triple beams

\begin{tabular}{lll}
$E$ & Steel's Young modulus $(G P a)$ & 210 \\
$\rho_{s}$ & Steel density $\left(\mathrm{kg} / \mathrm{m}^{3}\right)$ & 7800 \\
$h$ & Beam thikness $(\mathrm{m})$ & $1010^{-3}$ \\
$b$ & Beam width $(\mathrm{m})$ & $5010^{-3}$ \\
$L_{1}$ & Beam first length $(\mathrm{m})$ & 0.6 \\
$L_{2}$ & Beam second length $(\mathrm{m})$ & 0.5 \\
$L_{3}$ & Beam second length $(\mathrm{m})$ & 0.5 \\
$\nu$ & Steel's Poisson ratio & 0.3 \\
\hline
\end{tabular}

$$
\omega_{j}-\omega_{i} \gg 2 \times \xi_{i} \times \omega_{i}
$$

Namely, two modes $i$ and $j$ are decoupled if the pulsation difference between these two modes is much greater than the product of the pulsation and the damping coefficient of one mode multiplied by two. We focus on the case of neighboring modes so eigenfrequencies $\left(\omega_{i}=2 * \pi * f_{i}\right)$ must respect the following inequation :

$$
f_{j}-f_{i} \leq 2 \times \xi_{i} \times f_{i}
$$


We can thus define a theoretical limit frequency neighborhood as follows :

$$
f_{j \lim }=\left(1+2 \times \xi_{i}\right) f_{i}
$$

To investigate the effect of high modal density a study campaign has been performed. This study consists at evolving the parameters of the stiffness and keeping damping parameters unchangeable. The aim of changing the stiffness of the interfaces is to get neighboring modes, so that we can study the importance of this phenomena when applying the iterative energetic method to calculate damping. In this section, the used parameters are performed in table 3 .

Table 3: interfaces parametres evolution

\section{Damping}

Nonidentical and fixed damping $c_{i}$ $c_{1}=40 ; c_{2}=80 ; c_{3}=40$

$\cdots$

\section{Stiffness}

$$
\begin{aligned}
& \text { Identical and evolutive Stiffness } k_{i} \\
& \text { case } 1: k_{1}=k_{2}=k_{3}=110^{4} \\
& \text { case } 2: k_{1}=k_{2}=k_{3}=210^{4} \\
& \text { case } 3: k_{1}=k_{2}=k_{3}=410^{4} \\
& \ldots \\
& \text { case } \mathrm{N}: k_{1}=k_{2}=k_{3}=610^{5}
\end{aligned}
$$

For the first case damping results are presented in table 4. We get two triplets of neighboring modes, the first one is composed of modes $\{1,2,3\}$, and the second one is composed of modes $\{4,5,6\}$.

Table 4: Comparison of modal damping calculated using three methods

\begin{tabular}{lllllll}
\hline Modes $(\mathrm{Hz})$ & 1 & 2 & 3 & 4 & 5 & 6 \\
Frequenciesf $_{i}$ & 141.26 & 143.09 & 145.25 & 920.58 & 920.93 & 921.01 \\
$f_{i+1}-f_{i}$ & 1.83 & 2.17 & 775.33 & 0.35 & 0.08 & \\
$f_{l i m}$ & & 145.74 & 173.79 & & 923.13 & 938.43 \\
$\xi_{\nu}{ }^{\operatorname{vef}}(\%)$ & 0.79 & 5.36 & 16.53 & 0.06 & 0.47 & 1.48 \\
$\xi_{\nu}{ }^{\operatorname{ar} 2}(\%)$ & 0.88 & 5.64 & 16.14 & 0.07 & 0.50 & 1.45 \\
$\operatorname{Error}(\%)$ & 11.74 & 5.15 & 2.32 & 12.07 & 5.23 & 2.06 \\
\hline
\end{tabular}

It is found that the error increases when the eignemodes are more close. The error can exceed $10 \%$ when the frequency of neighbor modes becomes less than the theoretical limit frequency. For example the mode 4 depicts an error equal to $12.08 \%$ with a frequency spacing of $0.35 \mathrm{~Hz}$. $\left(f_{1}=920.59 \mathrm{~Hz}, f_{2}=920.93 \mathrm{~Hz}, f_{\text {lim }}=923.13 \mathrm{~Hz}\right.$ and $\left.f_{2} \prec f_{\text {lim }}\right)$. Figure 15 shows the evolution of the damping error of mode 4 (blue curve) and mode 5 (red curve) based on the difference between the frequencies of modes 4 and 5.

Each point of the two curves presents a test case. The frequency difference between Mode 4 and 5, presented in Table 4 and equal to $0.35 \mathrm{~Hz}$, shows the first point on the x-axis graph. The first point on the y-axis graph of the blue curve shows the relative error (\%) between damping calculated by the reference method and the energetic method EM2. The error is equal to $12.08 \%$. It is the same for the red curve which represents the error for mode 5 , the first point on the y-axes is an error of 5.24\%. Figure 15 illustrates also that when modes are more close then the damping error increases. The method EM2 may have some limitations for closed modes. Because, in case of high modal density it seems difficult to isolate a single mode, also because in this case the generalized damping matrix presents high non-diagonal terms, these terms affect the final results.

\section{Conclusion}

In this paper, the energetic method is presented and investigated for particular conditions to linear interface joints. The method is based on the ratio between the dissipated energy in the interfaces, and potential energy of the global structure. Firstly, the authors compared the localized damping in interfaces calculated by three methods. He aims at showing the importance of the mode projection by using a double beam system. Secondly, the effects of the localized damping level when localized viscous damping increases was investigated. Finally the influence of increasing modal density when calculation modal damping by the energetic method was studied. To do so, the academic structure of triple beams system was simulated. As a result, the energetic method is not valid when the system has a high modal density. 


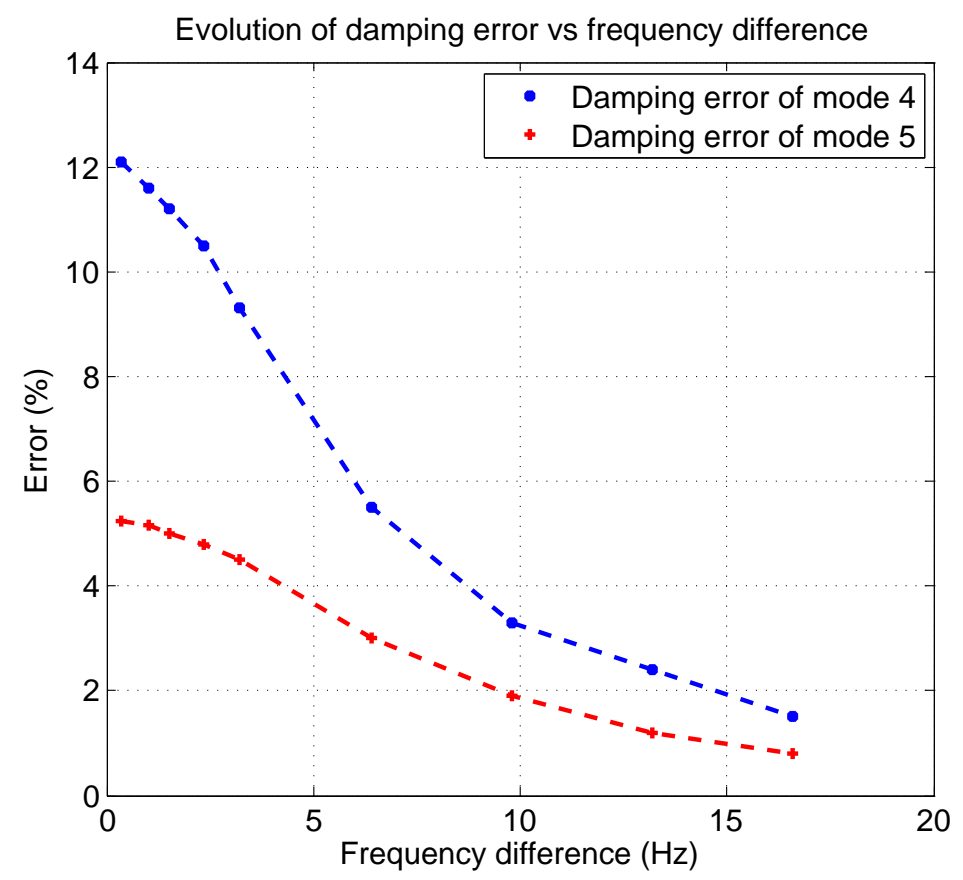

Figure 15: Evolution of damping errors vs frequency difference

\section{Acknowledgments}

Autors are grateful to the National Research Agency ANR for their financial support of this project (contract ANR 11).

\section{References}

[1] Bograd S., Reuss P., Schmidt A., Gaul L. and Mayer M. Modeling the dynamics of mechanical joints. Mechanical Systems and Signal Processing, Volume 25, Issue 8, 2801-2826, 2011.

[2] Gaul L., Roseira J., and Becker J. Structural damping with friction beams. Shock and Vibration, 15 3-4, 291-298, 2008.

[3] Ibrahim R. A. and Petit C. L. Uncertainties and dynamic problems of bolted joints and other fasteners. Journal of Sound and Vibration, 279, 857-936, 2005.

[4] Dion J. L., Chevallier G., Peyret N., Improvement of measurement techniques for damping induced by micro-sliding Mechanical Systems and Signal Processing, 34, 106-115, 2013.

[5] Caignot A., Ladeveze P., Neron D. and Durand J.-F., Virtual testing for the prediction of damping in joints. Engineering Computations, 5, 621-644.15, 2010.

[6] Geradin M., Rixen D. Mechanical vibrations. John Wiley, 1997.

[7] Adhikari S., Rates of change of eigenvalues ans eigenvectors in damped dynamic systems. AIAA Journal, 37(11), 1152-1158, 1999.

[8] Hasselman T. K., Modal coupling in lightly damped structures AIAA Journal, 11(2), 229-243, 1997 